\title{
Trends in the incidence of gastric cancer in Japan and their associations with Helicobacter pylori infection and gastric mucosal atrophy
}

\author{
Takanori Kobayashi ${ }^{1}$, Shogo Kikuchi ${ }^{1}$, Yingsong Lin $^{1}$, Kiyoko Yagyu ${ }^{1}$, Yuki Obata ${ }^{1}$, Atsushi Ogihara ${ }^{2}$, \\ Ayako Hasegawa ${ }^{3}$, Kazumasa Miki ${ }^{4}$, Eizo Kaneko ${ }^{5}$, Hiroshi Mizukoshi ${ }^{6}$, Tsuguo Sakiyama ${ }^{7}$, \\ and Hiroshi TENJIN ${ }^{7}$ \\ ${ }^{1}$ Department of Public Health, Aichi Medical University School of Medicine, 21 Karimata, Yazako, Nagakute-cho, Aichi 480-1195, Japan \\ ${ }^{2}$ Tokyo University of Science, Yamaguchi, Japan \\ ${ }^{3}$ Infectious Disease Surveillance Center, National Institute of Infectious Diseases, Tokyo, Japan \\ ${ }^{4}$ Division of Gastroenterology and Hepatology, Department of Internal Medicine (Ohmori), School of Medicine, Faculty of Medicine, \\ Toho University, Tokyo, Japan \\ ${ }^{5}$ First Department of Internal Medicine, Hamamatsu University School of Medicine, Hamamatsu, Japan \\ ${ }^{6}$ Tokyo Orimono Health Insurance Society Clinic, Tokyo, Japan \\ ${ }^{7}$ Clinic Attached to Kanto-Shin-Etsu Regional Taxation Bureau, Saitama, Japan
}

\begin{abstract}
Background. Although age-adjusted mortality from gastric cancer has been decreasing in Japan, the crude incidence of gastric cancer shows a slight increase.

Methods. We have observed trends in the incidence of gastric cancer by sex and 20-year age groups over the past two decades (1976-1996). Source data were obtained from the cancer statistics materials provided by the Research Group for Population-Based Cancer Registration in Japan. Simultaneously, we observed changes in the prevalence of Helicobacter pylori infection and in serological atrophy of the gastric mucosa, and compared the results with those involving changes in the incidence of gastric cancer.

Results. A slight decline was observed in all age groups over 40 years old, in both men and women, between 1986 and 1996. However, a marked decline in incidence was observed for those aged 20-39 years. The prevalence of $\boldsymbol{H}$. pylori infection declined in both sexes between 1989 and 1998. The frequency of serological atrophy of the gastric mucosa significantly declined in all age groups between 1989 and 1996, with young age groups experiencing a more marked decrease.

Conclusion. The marked decline in gastric cancer incidence observed in the young population will also begin to occur in the elderly population in the future.
\end{abstract}

Key words Gastric cancer incidence - Helicobacter pylori . Pepsinogen · Gastric mucosal atrophy

Offprint requests to: $\mathrm{S}$. Kikuchi

Received: March 3, 2004 / Accepted: August 6, 2004

\section{Introduction}

Despite a marked decline in the incidence of gastric cancer in many industrialized countries, gastric cancer is still the second most common cause of cancer-related deaths in Japan [1-4]. Based on regional cancer registrations, the incidence of gastric cancer in Japan in 1997 was 99318 (male, 66307 and female, 33011), accounting for $20.7 \%$ of the total cancer incidence in the same year [5]. The number of gastric cancer deaths in 1997 was 49739 (male, 32218 and female, 17521) based on vital statistics [6]. Age-adjusted mortality has been decreasing in both men and women, although crude mortality has not changed. Gastric cancer mortality is about half of the incidence, due to improved diagnostic and therapeutic techniques.

It is well known that Helicobacter pylori infection is one of the major risk factors for gastric cancer, and that low values of the pepsinogen (PG) I-to-II ratio can be a marker for atrophy of the gastric mucosa, as well as a marker for gastric cancer risk [7-10]. Therefore, the prevalence of $H$. pylori infection and the frequency of gastric mucosal atrophy may influence the incidence of gastric cancer.

In this study, we analyzed trends in the incidence of gastric cancer over the past two decades, by sex and 20 -year age groups. At the same time, we observed changes in the prevalence of $H$. pylori infection and in serological atrophy of the gastric mucosa, and compared them with changes in the incidence of gastric cancer. 


\section{Methods}

Cancer incidence figures in Japan are available from the Research Group for Population-Based Cancer Registration of the Ministry of Health, Labour, and Welfare, Japan, which issues annual cancer statistical data. We analyzed trends in the incidence of gastric cancer from 1976 to 1996 . The figures from 1985 to 1989 were acquired from the published data [11] that this research group had reported, and the pre-1984 data were from the group's website. The gastric cancer incidence in four 20 -year age groups $(20-39,40-59,60-79$, and 80 and over) from 1975 to 1996 was calculated using these data. Mortalities from gastric cancer in 1986 and 1996 were acquired from published data [6] from the Statistics and Information Department, Minister's Secretariat, Ministry of Health, Labour, and Welfare, Japan. To observe trends in gastric cancer incidence and its mortality, the 1996/1986 ratios of incidence and mortality were calculated by dividing the value in 1996 by the one in 1986 . In order to compare findings with the results for frequency of serological atrophy of the gastric mucosa, the 1996/ 1989 ratios of the incidence were also calculated.

For our analysis of the frequency of serological atrophy of the gastric mucosa, subjects were recruited from a workplace in the Kanto-shin-etsu area of Japan; 4486 of the subjects had participated in a health checkup program in 1989, and 4506 had participated in 1996. Serum PG I and serum PG II values were measured using residual sera from the health checkup programs in those 2 years. Measurements were carried out by immunoradiometric assay, with Pepsinogen I/II Riabead kits (Dainabot, Tokyo, Japan). When the serum PG I level was $70 \mathrm{ng} / \mathrm{ml}$ or less and the PG I/II ratio was 3.0 or less, it was defined as "serological atrophy" of the gastric mucosa [12].

To determine the prevalence of $H$. pylori, the subjects were selected from workers who belonged to a group of companies in the Tokyo area who were aged 50-59. Six hundred and seventy-six (male, 532; female, 144) of them took part in a general health checkup program in 1989, and 1916 (male, 1580; female, 336) took part in 1998. The sera in 1989 had been frozen for 10 years at $-30^{\circ} \mathrm{C}$, and those in $1998 \mathrm{had}$ been frozen for 1 year at $-30^{\circ} \mathrm{C}$ before the measurements. Serum H. pylori antibodies were measured using both the 1989 and 1998 sera. The measurements were carried out by enzymelinked immunosorbent assay (ELISA) with Pilika-Plate G Helicobacter II, produced by Biomerica (Newport Beach, CA, USA).

All statistical analyses were conducted using a commercial program for statistical analysis obtained from Halwin Gendai-Sugakusha (Kyoto, Japan). Our studies were approved by the Ethics Committee, Aichi Medical University School of Medicine.

\section{Results}

Figure 1 shows changes in gastric cancer incidence by sex in the 20-year age groups. A gradual decline in incidence was observed in all age groups, in both men and women, and the steepest decline was in the 20-39 age group. No clear change was observed before 1986, and a clear decline was observed only between 1986 and 1996, the trend of which was linear and statistically significant in each age group.

The 1996/1986 ratios of gastric cancer incidence for the age groups (Fig. 2) showed that the decline in younger age groups was more conspicuous than that in older age groups. The 1996/1989 ratios gave similar results, but the effect of age was not so clear in the age groups over 40 years.

On the other hand, the 1996/1986 ratios of gastric cancer mortality were in men, 0.516 for those aged 20-39; 0.667 for those aged 40-59; 0.759 for those aged $60-79$; and 0.923 for those aged 80 or more. Similarly, in women, the ratios were $0.445,0.689,0.652$, and 0.849 , respectively. The 1996/1986 ratios of gastric cancer mortality showed a trend of decline that was more conspicuous in younger age groups than in older ones.

Figure 3 shows the frequency of serological atrophy of the gastric mucosa in 1989 and 1996. During the 7year period, the frequency of serological atrophy of the gastric mucosa significantly declined in all age groups, with young age groups experiencing a more notable decrease.

Figure 4 shows the seroprevalence of $H$. pylori among those aged 50-59, in 1989 and 1998. The prevalence of $H$. pylori infection declined significantly in both sexes over the 9-year period of this study.

\section{Discussion}

Because the incidence of gastric cancer is low in people under 40 years of age, and because analysis by 20 -year groups can maintain the stability of data, we examined the trends in mortality and incidence by 20 -year age groups. A decrease in gastric cancer incidence was revealed, with those aged 20 to 40 showing the most rapid decrease, in both men and women. The decrease was observed only after 1986. Highly salted food intake, smoking, and drinking are enumerated as three major risk factors for gastric cancer, other than Helicobacter pylori infection and gastric mucosal atrophy. However, these three risk factors do not seem to explain the decrease sufficiently, for the following reasons. Firstly, although a change in the whole amount of salt intake does not necessarily correlate with a change in the dietary intake of highly salted foods, it is unlikely that the dietary intake of highly salted foods fell rapidly, because it 

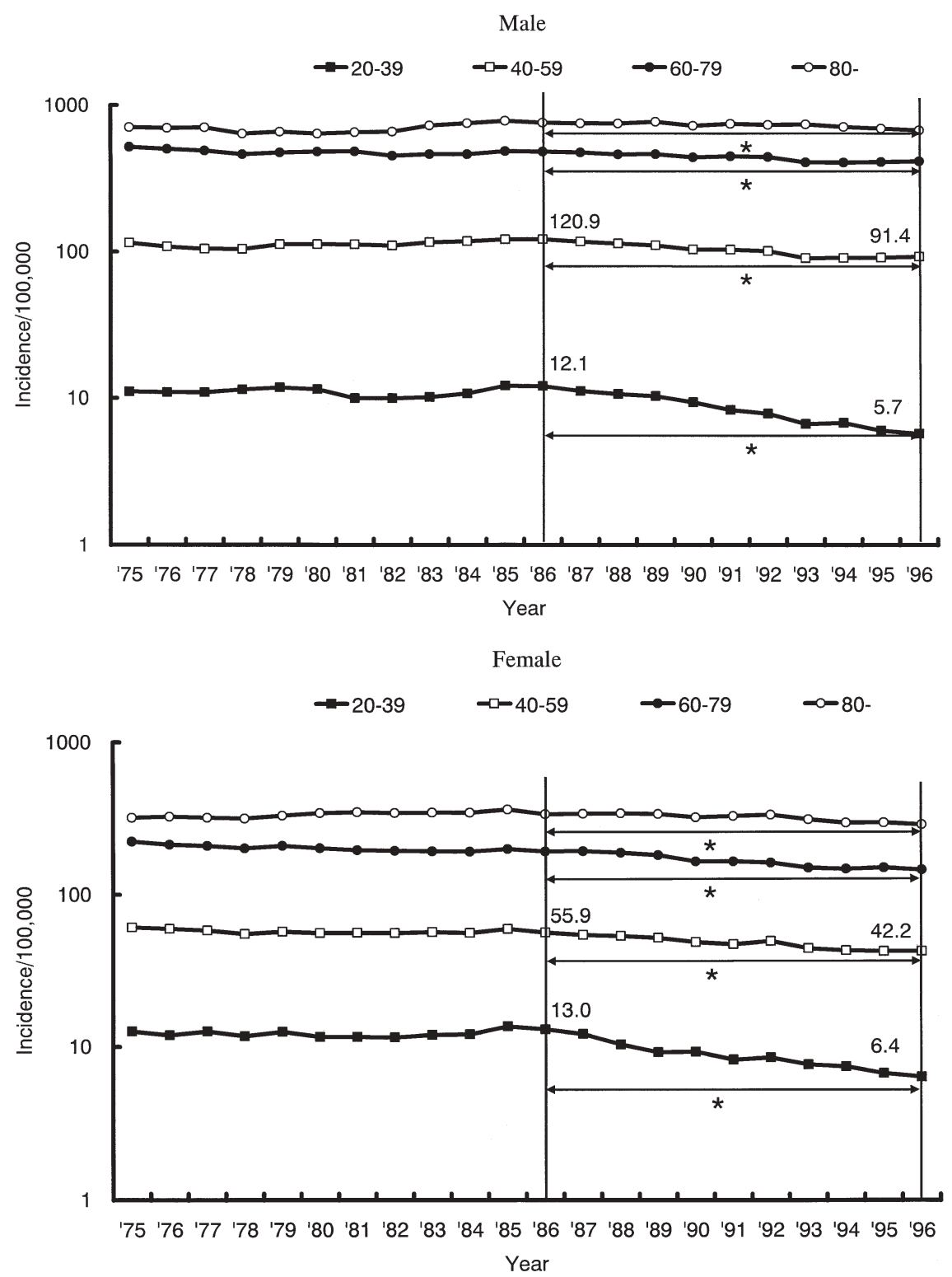

Fig. 1. Changes in gastric cancer incidence according to age are shown. A decline in incidence was observed in all 20-year age groups of both sexes between 1986 and 1996, and the decline in those aged 20-39 years was the steepest. $P$ value for trends, $<0.001$ has been shown that the amount of salt intake has almost leveled off, according to the National Nutrition Survey in Japan [13]. Secondly, the smoking rate has been decreasing in men and has leveled off in women, but is increasing in young women. The drinking trends have been similar to the smoking trends [14]. Therefore, these three factors do not seem to be major causes of the observed decline in gastric cancer incidence. Thus, among the possible factors contributing to the decrease, two in particular appear to be involved, i.e., changes in the prevalence of $H$. pylori infection, and the diminishing frequency of serological atrophy of the gastric mucosa.

\section{Changes in the prevalence of $\mathrm{H}$. pylori infection}

Numerous epidemiological and experimental studies using animals have shown that $H$. pylori infection is a causal risk factor for gastric cancer $[15,16]$. H. pylori infection occurs mostly in childhood [17-23] and continues for almost the entire life of the patient. Its overall prevalence is strongly correlated with socioeconomic factors, such as living conditions [24-26], water supply, and sewerage $[27,28]$, which may be especially important in developing countries. In many cross-sectional studies, an increase in the prevalence of $H$. pylori with age has been observed, for which there are two hypotheses: one is that new infections in the aging population increase the gradient of the prevalence; the other is that the gradient is influenced by a cohort effect, 


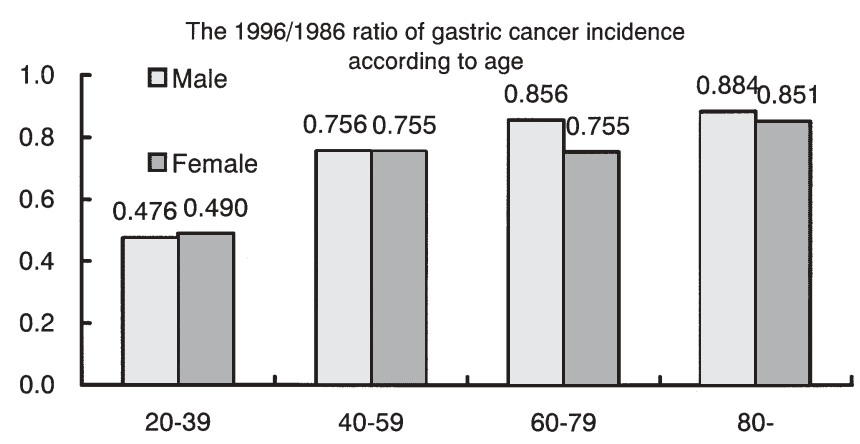

The 1996/1989 ratio of gastric cancer incidence according to age

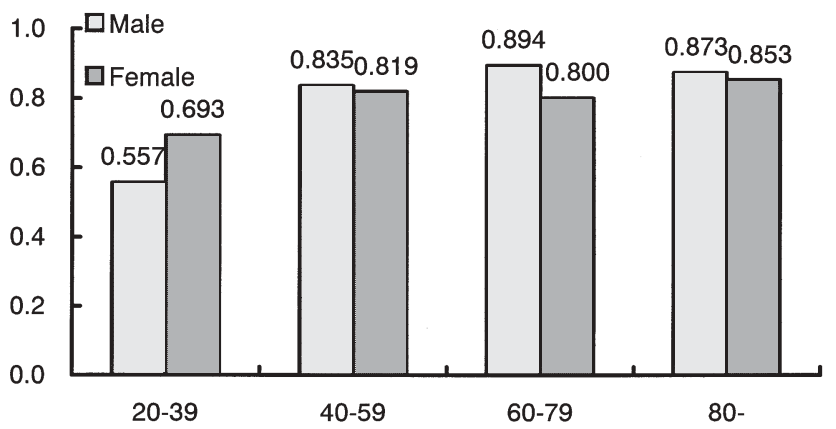

Fig. 2. The 1996/1986 ratios of gastric cancer incidence (over a 10-year period) for each age group showed that the decline was more conspicuous in young age groups than in old age groups. Similar results were observed in the 1996/1989 ratios (over a 7-year period), but the effect of age was not so clear among the age groups aged over 40 years

reflecting more widespread transmission contemporaneous with the childhood years of earlier birth cohorts $[19,29,30]$.

In order to clarify which hypothesis is correct, we compared the prevalence of $H$. pylori between the subjects aged 50-59 years in 1989 and those aged 50-59 years in 1998 in a workers' group and found a decline in seroprevalence over the 9-year period. Although the subjects were only from the Tokyo region, another study with subjects from other areas throughout Japan (Nagano, Niigata, Gunma, Toyama, Shizuoka, Mie, and Miyagi prefectures) has reported the same results [31]. Although these results may not be sufficiently representative of conditions in Japan as a whole, no contradictory results have been found, as far as we can discover, suggesting that the cohort effect of improved hygiene exerts a greater influence on the overall rate of prevalence than new infections with aging [32-34].

Although hygiene conditions in Japan were poor just after World War II, they have gradually improved since about 1950, which seems to support the conclusion mentioned above. If the prevalence of $H$. pylori mainly depends on hygiene conditions in childhood, a decline in $H$. pylori prevalence owing to improved hygiene conditions must occur first in the younger population. These explanations seem to be consistent with the observed decrease in gastric cancer incidence in that young population.

\section{Frequency of serological atrophy of the gastric mucosa}

It has been established that people with atrophic gastritis have a high risk for gastric cancer [26,35]. Previous studies have shown that serum PG values were strongly associated with gastric cancer [36]. In people under 40 years of age, the PG II level, as well as the PG I/II ratio, showed a strong association with the risk for gastric cancer [37]. Among those over 40, a strong association was also observed between the gastric cancer risk and serological atrophy of the gastric mucosa [10]. In addition, the relation between atrophic gastritis and gastric cancer has been confirmed by endoscopic studies [38].

Serum PGs are markers for atrophic gastritis and gastric mucosal atrophy. A low serum PG I level and a low PG I/II ratio are related to atrophy of the gastric mucosa, as well as being related to inflammation of the gastric mucosa $[39,40]$. Therefore, we defined low values for serum PGs (serum PG I level of $70 \mathrm{ng} / \mathrm{ml}$ or less and PG I/II ratio of 3.0 or less) as serological atrophy. These low levels are also related to the risk for gastric cancer and are sometimes used in screening programs for gastric cancer [12]. If gastric mucosal atrophy is related to the risk for gastric cancer, a consistent change in the prevalence of gastric mucosal atrophy might be expected in conjunction with the observed decline in the gastric cancer incidence. We observed a change in the frequency of serological atrophy of the gastric mucosa over a 7-year period, and compared it with the change in gastric cancer incidence over the same period.

The frequency of serological atrophy of the gastric mucosa decreased over that 7-year period. The marked decline in frequency of the atrophy cannot be explained without considering the possibility of reversible change in serological atrophy. Indeed, $63 \%$ of the subjects experienced an increase or no change in the PG I/II ratio over the 7 -year period $[39,40]$, indicating that serological atrophy of the gastric mucosa is reversible.

The decline in young age groups was more conspicuous than that in older groups. Although the subjects did not necessarily represent the Japanese population as a whole, they were, in fact, from relatively wide spread areas: Niigata, Nagano, Gunma, Tochigi, Ibaraki, Saitama, and Tokyo.

So far, many studies have noted the prevalence of gastric mucosal atrophy, but few have examined the 
Male

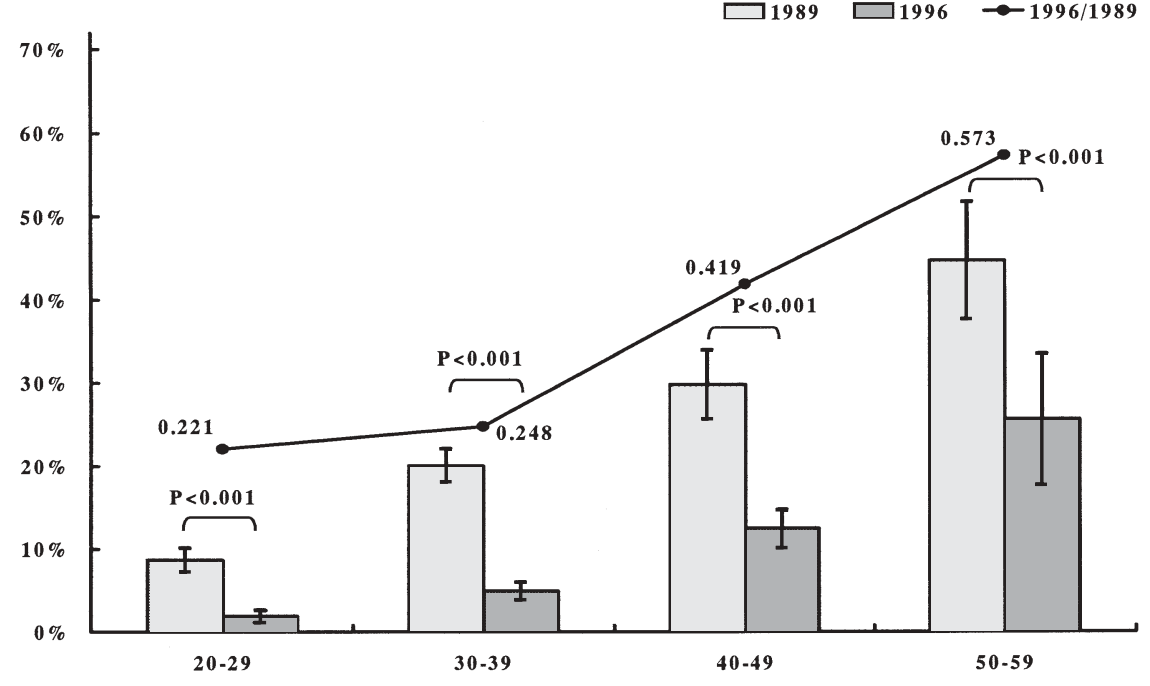

Female

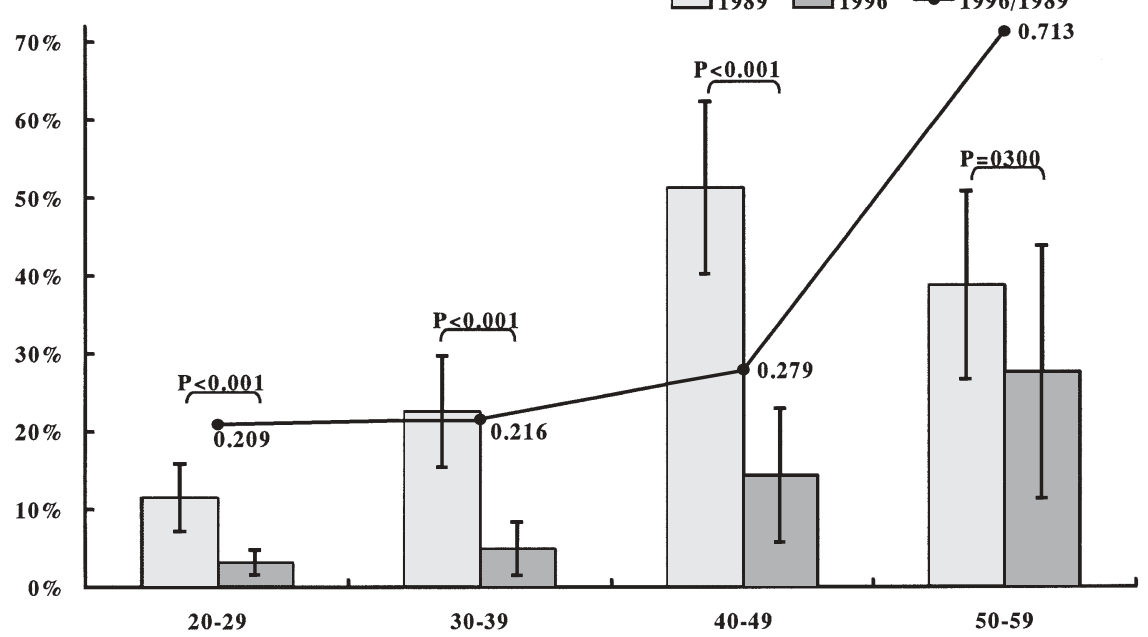

Changes in prevalence of Helicobacter pylori infection among those aged 50-59

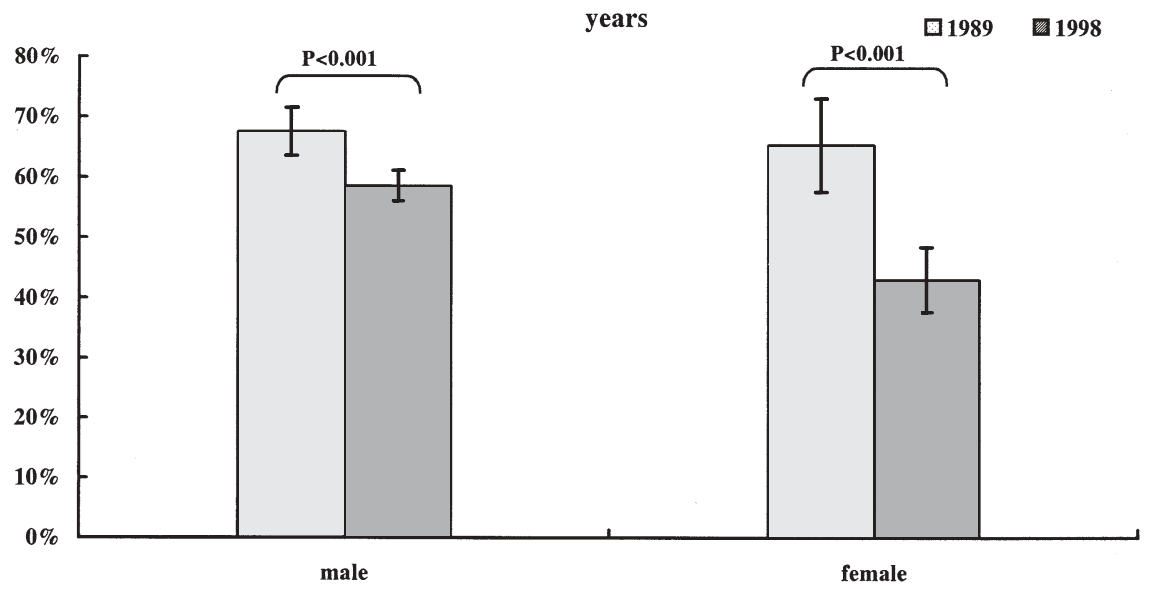

Fig. 3. Frequencies of serological atrophy of the gastric mucosa in 1989 and in 1996. During the 7-year period, the frequency of serological atrophy of the gastric mucosa significantly declined in all age groups, with younger age groups showing a particularly marked decrease
Fig. 4. The prevalence of Helicobacter pylori infection declined in both sexes between 1989 and 1998 
chronological changes in PG I and II values over a period of several years as our study has. We could find only one study [41] that showed the same trends in serological atrophy as our study did. A large-scale study of Japanese subjects reported that very few cases of atrophy of the gastric mucosa were observed among those without $H$. pylori infection [42], further confirming that a decrease in $H$. pylori prevalence may result in a decrease in gastric mucosal atrophy. Studies reporting a decrease in $H$. pylori prevalence over the years may be considered as indicating a concomitant decrease in gastric mucosal atrophy.

The prevalence of gastric mucosal atrophy seems to have clearly decreased in Japan, along with the prevalence of $H$. pylori infection, with the decrease in the younger population being more conspicuous. Although there may be some limitations to the conclusions discussed above, these findings would seem to explain the decline we observed in gastric cancer incidence. The change in frequency of serological atrophy of the gastric mucosa was more rapid compared with the change in gastric cancer incidence, and this may have occurred because there was a time lag between the beginning of gastric cancer in the atrophic mucosa and the clinical diagnosis of the cancer when it has developed.

\section{Future incidence of gastric cancer}

In Japan, there seems to be a decreasing birth cohort effect for $H$. pylori infection, as well as for serological atrophy of the gastric mucosa. The decreasing birth cohort effect is expected to continue in the future. In the observation period of this study, a decline in the incidence of gastric cancer was observed, and it was most marked in those aged 20-39 years. It is expected that the marked decline in the incidence of gastric cancer in the younger population may extend to the older population in the future, as the young population with a low prevalence of $H$. pylori infection gets older.

Acknowledgments We thank the Research Group for Population-Based Cancer Registration, of the Ministry of Health, Labour, and Welfare of Japan, for kindly providing us with their data on cancer statistics. We express special thanks to Dr. Hiroshi Kaneko for kindly providing us with useful comments. We are also indebted to Ms. Maya Oikawa and Ms. Mayumi Endo for their valuable technical assistance in making the graphs.

\section{References}

1. Axon A. Gastric cancer and Helicobacter pylori. Aliment Pharmacol Ther 2002;16 (Suppl 4):83-8.
2. Charles SF, Robert JM. Gastric carcinoma. N Engl J Med 1995; 333:32-41.

3. Ahlgren JD. Gastrointestinal malignancies. Prim Care 2001;28: 647-60.

4. Whelan SL. Quality control of international cancer incidence data. Health Rep 1993;5:73-9.

5. The Research Group for Population-based Cancer Registration in Japan. Cancer incidence and incidence rates in Japan in 1997: estimates based on data from 12 population-based cancer registries. Jpn J Clin Oncol 2002;32:318-22.

6. Statistics and Information Department, Minister's Secretariat, Ministry of Health, Labour, and Welfare. Vital statistics of Japan 1997 (in Japanese). Tokyo. pp. 138-9.

7. Uemura N, Okamoto S, Yamamoto S, Matsumura N, Yamaguchi S, Yamakido M, et al. Helicobacter pylori infection and the development of gastric cancer. N Engl J Med 2001;345:784-9.

8. Forman D, Newell DG, Fullerton F, Yarnell JW, Stacey AR, Wald N, et al. Association between infection with Helicobacter pylori and risk of gastric cancer. BMJ 1991;302:1302-5.

9. Kikuchi S. Epidemiology of Helicobacter pylori and gastric cancer. Gastric Cancer 2002;5:6-15.

10. Miki K, Ichinose M, Kawamura N, Matsushima M, Ahmad HB, Kimura M, et al. The significance of low serum pepsinogen levels to detect stomach cancer associated with extensive chronic gastritis in Japanese subjects. Jpn J Cancer Res 1989;80:111-4.

11. Research Group for Population-based Cancer Registration in Japan. Cancer incidence in Japan, 1985-89: re-estimation based on data from eight population-based cancer registries. Jpn J Clin Oncol 1998;28:54-67.

12. Watanabe Y, Kurata JH, Mizuno S, Mukai M, Inokuchi H, Miki $\mathrm{K}$, et al. Helicobacter pylori infection and gastric cancer. A nested case-control study in a rural area of Japan. Dig Dis Sci 1997;42: 1383-7.

13. Ministry of Health, Labour, and Welfare, Japan. The National Nutrition Survey in Japan, 2001 (in Japanese). Tokyo. p. 156.

14. Health and Welfare Statistics Association. Problems associated with health. J Health Welf Stat 2003;50:81-6.

15. Parsonnet J, Friedman GD, Vandersteen DP, Chang Y, Vogelman JH, Orentreich N, et al. Helicobacter pylori infection and the risk of gastric carcinoma. N Engl J Med 1991;325:1127-31.

16. Peterson WL. Helicobacter pylori and gastric adenocarcinoma. Aliment Pharmacol Ther 2002;16 (Suppl 1):40-6.

17. Miyaji H, Azuma T, Ito S, Abe Y, Gejyo F, Hashimoto N, et al. Helicobacter pylori infection occurs via close contact with infected individuals in early childhood. J Gastroenterol Hepatol 2000;15: 257-62.

18. Imrie C, Rowland M, Bourke B, Drumm B. Is Helicobacter pylori infection in childhood a risk factor for gastric cancer? Pediatrics 2001:107:373-80.

19. Banatvala N, Mayo K, Megraud F, Jennings R, Deeks JJ, Feldman RA. The cohort effect and Helicobacter pylori. J Infect Dis 1993;168:219-21.

20. Cullen DJE, Collins BJ, Christiansen KJ, Epis J, Warren JR, Surveyor I, et al. When is Helicobacter pylori infection acquired? Gut 1993;34:1681-2.

21. Mitchell HM, Li YY, Hu PJ, Liu Q, Chen M, Du GG, et al. Epidemiology of Helicobacter pylori in southern China: identification of early childhood as the critical period for acquisition. J Infect Dis 1992;166:149-53.

22. Kikuchi S, Kurosawa M, Sakiyama T. Helicobacter pylori risk associated with sibship size and family history of gastric diseases in Japanese adults. Jpn J Cancer Res 1998;89:1109-12.

23. Ueda M, Kikuchi S, Kasugai T, Shunichi T, Miyake C. Helicobacter pylori risk associated with childhood home environment. Cancer Sci 2003;94:914-8.

24. Webb PM, Knight T, Greaves S, Wilson A, Newell DG, Elder J, et al. Relation between infection with Helicobacter pylori and living conditions in childhood: evidence for person to person transmission in early life. BMJ 1994;308:750-3. 
25. Sipponen P, Kousnen TU, Samloff IM, Heinonen OP, Siurala M. Rate of Helicobacter pylori acquisition among Finnish adults. Scand J Gastroenterol 1996;31:229-32.

26. Kurosawa M, Kikuchi S, Inaba Y, Ishibashi T, Kobayashi F. Helicobacter pylori infection among Japanese children. J Gastroenterol Hepatol 2000;15:1382-5.

27. Krumbiegel P, Boeckler D, Lehmann I, Jorks S, Fritz G, Babel W, et al. Is water a source of $H$. pylori infection in central Europe (abstract)? Gut 2000;Suppl 1:46.

28. Olmos JA, Rios H, Higa R and the Argentinean Hp Epidemiologic Study Group. Prevalence of Helicobacter pylori infection in Argentina: results of a nationwide epidemiologic study. J Clin Gastroenterol 2000;31:33-7.

29. Suerbaum S, Michetti P. Helicobacter pylori infection. N Engl J Med 2002;347:1175-86.

30. Goodman KJ, Correa P, Tengana Aux HJ, Ramirez H, DeLany JP, Guerrero Pepinosa O, et al. Helicobacter pylori infection in the Colombian Andes: a population-based study of transmission pathways. Am J Epidemiol 1996;144:290-9.

31. Fujisawa T, Kumagai T, Akamatsu T, Kiyosawa K, Matsunaga Y. Changes in seroepidemiological pattern of Helicobacter pylori and hepatitis A virus over the last 20 years in Japan. Am J Gastroenterol 1999;94:2094-9.

32. Robert R, Kuipers EJ, Buitenwerf J, van Uffelen C, Meuwissen SGM, van Kamp GJ, et al. Helicobacter pylori and the birth cohort effect: evidence of a continuous decrease of infection rates in childhood. Am J Gastroenterol 1997;92:1480-2.

33. Last JM. A dictionary of epidemiology. 4th ed. New York: Oxford University Press; 2001. p. 76.
34. van Zanten SJO, Pollak PT, Best LM, Bezanson GS, Marrie T. Increasing prevalences of Helicobacter pylori infection with age: continuous risk of infection in adults rather than cohort effect. J Infect Dis 1994;169:434-7.

35. Kitahara F, Shimazaki R, Sato T, Kojima Y, Morozumi A. Severe atrophic gastritis with Helicobacter pylori infection and gastric cancer. Gastric Cancer 1998;1:118-24.

36. Tsubono Y, Hisamichi S. Screening for gastric cancer in Japan. Gastric Cancer 2000;3:9-18.

37. Kikuchi S, Wada O, Miki K, Nakajima T, Nishi T, Kobayashi O, et al. Serum pepsinogen as a new marker for gastric carcinoma among young adults. Cancer 1995;75:2789-93.

38. Kato I, Tominaga S, Ito Y, Kobayashi S, Yoshii Y, Matsuura A, et al. A prospective study of atrophic gastritis and stomach cancer risk. Jpn J Cancer Res 1992;83:1137-42.

39. Kikuchi S, Kurosawa M, Sakiyama T, Tenjin H, Miki K, Wada O, et al. Long-term effect of Helicobacter pylori infection on serum pepsinogens. Jpn J Cancer Res 2000;91:471-6.

40. Kikuchi S, Kurosawa M, Sakiyama T, Tenjin H. Long-term effect of smoking on serum pepsinogen values. J Epidemiol 2002;12: 351-6.

41. Kinoshita Y, Kawanami C, Kishi K, Nakata H, Seino Y, Chiba T. Helicobacter pylori independent chronological change in gastric acid secretion in the Japanese. Gut 1997;41:452-8.

42. Asaka M, Sugiyama T, Nobuta A, Kato M, Takeda H, Gramam DY. Atrophic gastritis and intestinal metaplasia in Japan: results of a large multicenter study. Helicobacter 2001;6:294-9. 